\title{
Multiphase structural models and hyperpolarizability calculations explain second-order nonlinear optical properties of stilbazolium ions
}

Christopher M. Ashcroft, Jacqueline M. Cole, Tze-Chia Lin, SeungChul Lee, Lorraine A. Malaspina, and O-Pil Kwon

\section{Published version information}

Citation: CM Ashcroft et al. "Multiphase structural models and hyperpolarizability calculations explain second-order nonlinear optical properties of stilbazolium ions." Phys Rev Materials 4, no. 11 (2020): 115203.

DOI: $10.1103 /$ PhysRevMaterials.4.115203

This version is made available in accordance with publisher policies. Please cite only the published version using the reference above. This is the citation assigned by the publisher at the time of issuing the APV. Please check the publisher's website for any updates. 


\title{
Multiphase structural models and hyperpolarizability calculations explain second-order nonlinear optical properties of stilbazolium ions
}

\author{
Christopher M. Ashcroft, ${ }^{1}$ Jacqueline M. Cole $\odot,{ }^{1,2,3,4, *}$ Tze-Chia Lin, ${ }^{1}$ Seung-Chul Lee, ${ }^{5}$ \\ Lorraine A. Malaspina, ${ }^{6}$ and O-Pil Kwon ${ }^{5}$ \\ ${ }^{1}$ Cavendish Laboratory, Department of Physics, University of Cambridge, J. J. Thomson Avenue, Cambridge, CB3 OHE, United Kingdom \\ ${ }^{2}$ ISIS Neutron and Muon Source, Rutherford Appleton Laboratory, Harwell Science and Innovation Campus, \\ Didcot, Oxfordshire, OX11 OQX, United Kingdom \\ ${ }^{3}$ Department of Chemical Engineering and Biotechnology, University of Cambridge, West Cambridge Site, \\ Philippa Fawcett Drive, Cambridge, CB3 OAS, United Kingdom \\ ${ }^{4}$ Argonne National Laboratory, 9700 South Cass Avenue, Lemont, Illinois 60439, USA \\ ${ }^{5}$ Department of Molecular Science and Technology, Ajou University, Suwon 443749, South Korea \\ ${ }^{6}$ Institute of Inorganic Chemistry and Crystallography, University of Bremen, Leobener Strasse 7, 28359 Bremen, Germany
}

(Received 19 May 2020; accepted 14 October 2020; published 24 November 2020)

\begin{abstract}
The stilbazolium-based organic salt, DAST, is probably the most well-known commercial organic non-linear optical (NLO) material. Chemical derivatives of DAST have therefore been designed to engender even better performing NLO materials; the DAST-derivative, BP3, is one success in this regard. This paper provides a proof-of-principle in the use of structure factors, $F$, to determine the effects of the crystalline environment on intramolecular charge transfer (ICT), and thus the second-order NLO response of such molecules. A concerted experimental and computational approach is adopted. In particular, the application of the relatively new x-ray wave function refinement method, which tempers theoretical calculations with experimentally derived structure factors to yield an isolated molecule influenced by crystal-field forces, enables the impact of those crystal-field forces on ICT to be established. This study employs high-resolution x-ray diffraction data for its experimental component, this resolution being necessary given the marked challenges that are associated with the complicated interionic interactions within an organic salt. The results presented pinpoint the molecular-scale features that afford BP3 better second-order NLO prospects than DAST, laying the foundations for the molecular engineering of DAST derivatives that are better tailored to optimize NLO function.
\end{abstract}

DOI: 10.1103/PhysRevMaterials.4.115203

\section{INTRODUCTION}

\section{A. Molecular origins of second-order NLO properties in organic chromophores}

Organic nonlinear optical (NLO) chromophores have attracted much attention across a wide range of optical and optoelectronic applications owing to their large NLO responses and ultrafast response times. While their inorganic counterparts rely on the displacement of their lattice components (atoms or ions) to gain optical nonlinearity, the NLO effects in organic chromophores arise primarily from the molecular charge-transfer excitations induced by the interactions between external electromagnetic fields and the delocalized $\pi$-conjugated electrons of the molecules. Such organic molecules traditionally possess a donor- $\pi$-acceptor $(\mathrm{D}-\pi-\mathrm{A})$ architecture with high levels of intramolecular charge transfer (ICT). Such charge transfer is the main driving force for the NLO activity of such molecules $[1,2]$. Consequently, the individual chromophore of an organic NLO compound can be seen as the core intramolecular chargetransfer unit for various NLO processes. Over the last few

\footnotetext{
*Corresponding author: jmc61@ @am.ac.uk
}

decades, numerous structure-function relationships have been established for organic materials that exhibit second-order NLO effects [such as second harmonic generation (SHG)] [3]; these have since become the basis for the rational and systematic molecular design of new organic NLO chromophores.

\section{B. Organic salts as NLO chromophores: ionic and interionic considerations}

For nonionic organic chromophores, it can be generally assumed that their intermolecular interactions are weak. Therefore, the macroscopic (bulk) second-order NLO susceptibilities $\left(\chi^{2}\right)$ of these materials can be estimated by the orientational superposition of the molecular-scale secondorder NLO susceptibilities, i.e., $\beta$, which is known as the first-order molecular hyperpolarizability. However, this assumption, which is based on the oriented-gas model [4], cannot be used for ionic organic chromophores, such as stilbazolium-based compounds. Yet, such organic salts are particularly important in the NLO field. For instance, the stilbazolium-based chromophore, 4- $N, N$-dimethylamino- $N$ methyl-4-stilbazolium tosylate, DAST [5,6] (Fig. 1, left), is one of few organic molecules to have demonstrated longstanding commercial prospects for second-order NLO 


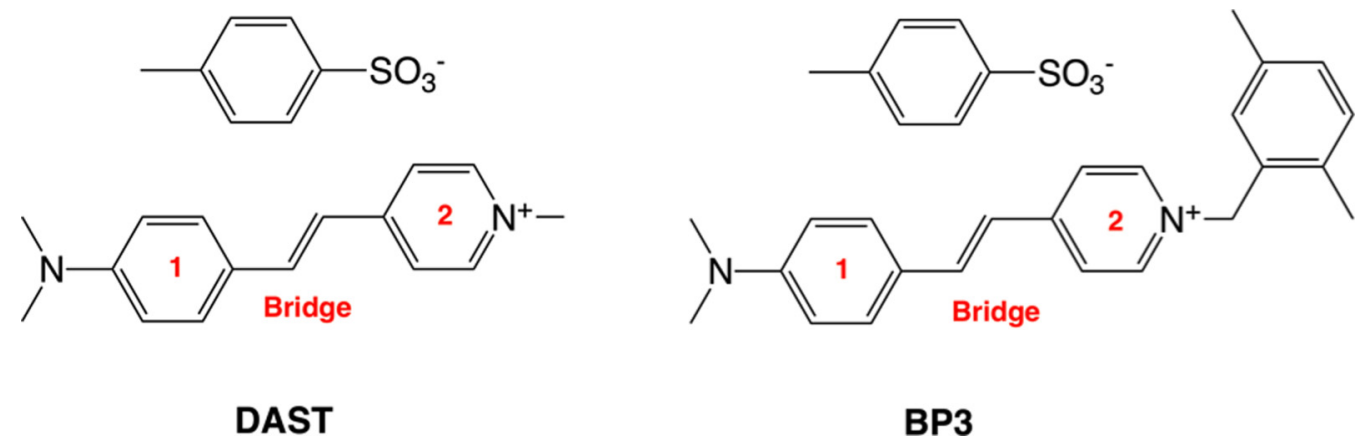

FIG. 1. Chemical structures of DAST and BP3 drawn in their default canonical representations.

applications [7-9] such as $\mathrm{THz}$ generation and, electrooptic single crystalline waveguides, to name but two. A range of DAST derivatives has been developed with the prospect that they might afford even better secondorder NLO prospects. Structure-property relationships that help decode the molecular-scale origins of such derivatives stand to offer materials-by-design strategies, which could be used to systematically tackle this materials discovery goal.

Compared to nonionic organic chromophores, there are more types of intermolecular (interionic) interactions in ionic organic chromophores that could influence the molecularscale origins of their NLO activity; for example, those emanating from van der Waals forces, electrostatic (Coulombic) forces, or hydrogen-bonding, which need to be considered carefully for an ionic organic chromophore. Moreover, since organic salts contain anions and cations, all combinations of cation $\cdots$ anion, cation $\cdots$ cation, and anion $\cdots$ anion interactions need to be assessed. Furthermore, the NLO output of an organic salt will be influenced by the relative structural arrangement of each type of ion with respect to each other within the crystal lattice.

Cole et al. [10] have shown that DAST (Fig. 1, left) exhibits a range of significant interionic interactions in its crystalline state, wherein the presence of certain combinations of cation ...anion interactions in DAST derivatives could diminish their molecular hyperpolarizability, $\beta$. In particular, the coexistence of $\mathrm{C}-\mathrm{H} \cdots \mathrm{O}$ and $\mathrm{C}-\mathrm{H} \cdots \mathrm{C}(\pi)$ hydrogen-bonds between cations and anions in DAST derivatives have been demonstrated to have a particularly deleterious effect on their $\beta$ values [10]. This led Cole et al. to advocate that DAST derivatives, which are void of such cation $\cdots$ anion interactions, could herald better second-order NLO prospects than DAST. For example, $N, N$-dimethylamino- $N^{\prime}-2,5$ - dimethylbenzylstilbazolium $p$-toluenesulfonate, BP3 [10,11] (Fig. 1, right), is void of cationic $\cdots$ anionic $\mathrm{C}-\mathrm{H} \cdots \mathrm{C}(\pi)$ hydrogen-bonds; this appears to be due to its 2,5-dimethylbenzyl moiety, which is responsible for an overall bent-shaped cation and results in larger cation $\cdots$ anion separations in BP3 than in DAST [10,12]. DAST and BP3 also crystallize in different space groups (Cc and $\mathrm{P} 2{ }_{1}$, respectively) [10]. Nevertheless, cation - . anion interactions in both DAST and BP3 essentially lie in crystallographic planes that are perpendicular to interleaving sheets of anion $\cdots$ anion and cation $\cdots$ cation interactions [13].

\section{Scope of paper}

We present a study that builds up the ionic-salt structures of DAST and BP3 step-by-step and determines their hyperpolarizabilities at each step. We explore the nature of their interionic interactions and assess how these affect their intraionic charge transfer and thus their second-order NLO responses. Starting from structural models of isolated DAST and BP3 ions, we assemble their crystal lattice environment in four key stages, while studying the structural and NLO modulations of the cation $\cdots$ anions as a consequence of these piece-meal changes. Our starting point is a negative pointcharge model that is similar to that employed by Kim et al. [12], except that our model is based on atomic coordinates from experimental diffraction data; the results indicate that cation...anion interactions need to be considered in greater detail (results are presented in the Supplemental Material [14]). From there, the crystalline environment of DAST and BP3 is built up piecewise via three distinct steps that employ the models and data described in Fig. 2. The first comprises an isolated cation created by $a b$ initio calculations using density functional theory (DFT) to which is added its nearestneighbor anion [Fig. 2(a)]; this model realizes the impact of the specific cation $\cdots$ anion interaction on the ICT of each organic salt. Models of their electronic structures, that represent an isolated cation and anion but which are subjected to crystal field forces, are then developed using x-ray wave function refinements (XWRs) [Fig. 2(b)]. Bond-length-alternation (BLA) analyses [3,15] performed on these XWR models are compared with cognate BLA results on the ion-pair in vacuum, revealing the influence of crystal field forces upon the ICT of each ionic salt. A multipolar structural analysis of x-ray diffraction data on DAST and BP3 is then carried out, to model these organic salts within their crystal lattice environment [Fig. 2(c)]. To this end, BLA analysis is first applied to these multipolar structural models to distinguish the effect of both crystal field forces and interionic interactions on their ICT and thus second-order NLO response. Electronic moments from these multipolar structural models are then used to derive solid-state values of $\beta$, using an approach based on Robinson $[16,17]$. These $\beta$ values are compared with those from gas-phase calculations using DFT, and with reference solution-state $\beta$ values of DAST [18] and BP3 [10] obtained from HRS experiments [19]. This multiphase determination of $\beta$ reveals a substantially smaller molecular-scale secondorder NLO response in DAST and BP3 in the crystalline 


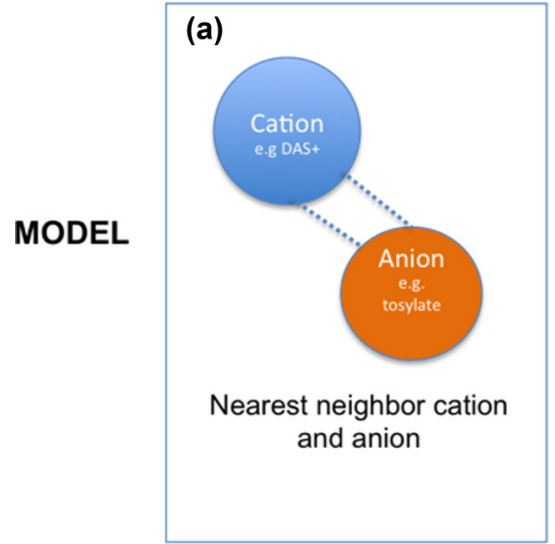

Theoretical structure factors generated a DFTderived wavefunction of the ionic system, using a super cell of $a=b=c=30 \AA$
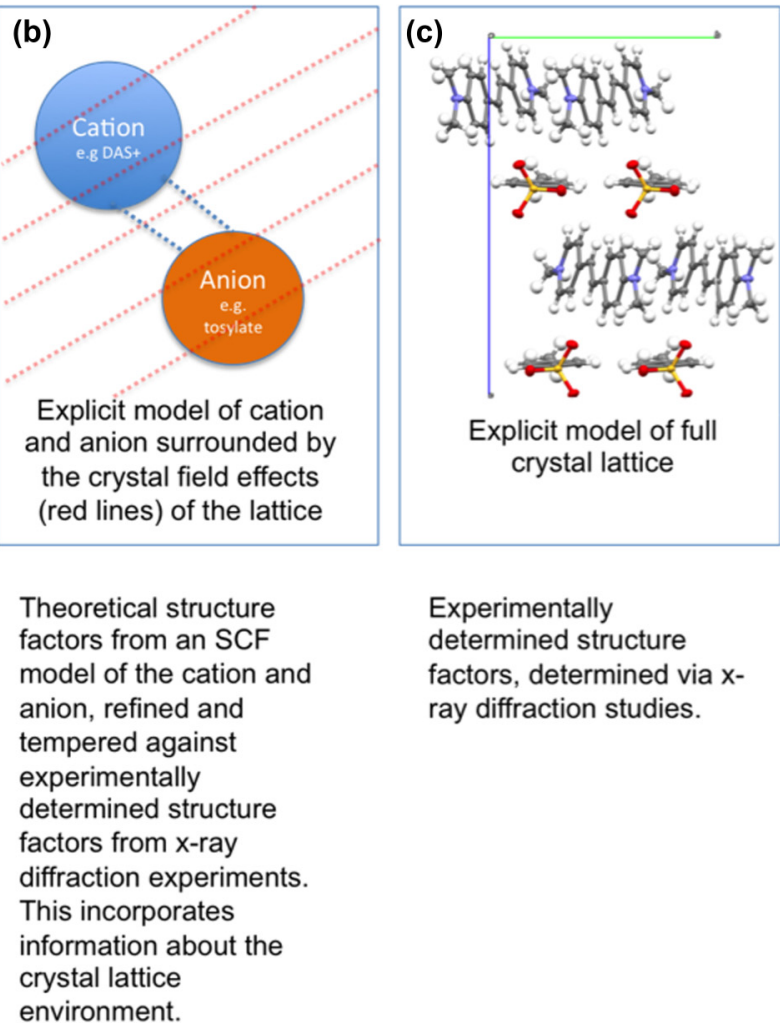

\section{Experimentally}

determined structure

factors, determined via $x$ -

ray diffraction studies.

FIG. 2. An illustration of steps 2, 3, and 4 of the four-step process by which structural models of DAST and BP3 are built up in order to determine how the ions are influenced by their crystal environment.

state; these results also show that $\beta$ in DAST progressively decreases from the gas to the solid phase. These observations are thence rationalized to elucidate intraionic and interionic structure-property relationships in DAST and BP3 that control their second-order NLO function. A validation of these models was explored and this is presented in the Supplemental Material [14,20-30]. We show that our findings validate the models, which in turn demonstrates the validity of constructing them for a vast range of prospective NLO materials. We conclude that these model constructs herald a predictive power in molecular design strategies that aim to discover new NLO materials. Full details regarding the experimental and computational methods used throughout this study are outlined in the Supplemental Material [4,8,14,31-56].

\section{RESULTS AND DISCUSSION}

A range of structural models of DAST and BP3 were built up ion-by-ion, initially using DFT to calculate various types of ionic clusters. These provided important checks to verify previous work on calculations involving cation...anion, cation $\cdots$ cation, and anion $\cdots$ anion interactions. Our results, detailed in the Supplemental Material [14] corroborate previous findings that anion $\cdots$ anion interactions in DAST and BP3 have negligible effect on their second-order NLO response [56], while cation $\cdots$ cation interactions in DAST and BP3 promote ICT, providing an overall positive contribution to the ensuing NLO activity of the chromophores [13]. The nature of cation $\cdots$ anion interactions presents a much more complex situation. For example, a previous structural study on crystalline DAST and BP3 suggested that cation $\cdots$ anion interactions could be responsible for either an enhancement or reduction of $\beta$ upon crystallization, depending on their exact nature [10]. Such interactions are thus the focus of the electronic structure models described in the next two sections. Studies were also undertaken to verify the findings of Kim et. al. [12]. These results are presented in the Supplemental Material [14].

\section{A. Overarching structural features of cations of DAST and BP3}

Figure 3 presents a cross comparison of bond lengths for the cations of both DAST and BP3 across all structural models: (a) (i) isolated cation; (ii) isolated cation with nearest neighbor anion interactions; (b) cation and anion under the influence of crystal field effects; (c) cations within their full crystal lattice environment. There are certain overarching similarities between DAST and BP3 as anticipated. For example, the pyridinium ion in ring 2 of both chromophores acts as the electron acceptor, while the ethylamino group adjacent to ring 1 is the electron donor. Rings 1 and 2 exhibit distinct quinoidal character; $c f$. the two shorter bond lengths for $\mathrm{C}(13)-\mathrm{C}(14)$, $\mathrm{C}(11)-\mathrm{C}(10)$ of ring 1 and $\mathrm{C}(5)-\mathrm{C}(6), \mathrm{C}(3)-\mathrm{C}(2)$ of ring 2 . The intervening bridge consists of an alternating single-doublesingle bonding chain, as indicated by the bond lengths across all structural models.

While the D- $\pi$-A motifs are the same for BP3 and DAST, BP3 naturally differs from DAST in that its cation contains an additional 1,2,4-trimethylbenzene moiety. The primary contribution of this moiety is to impart a steric influence on the 


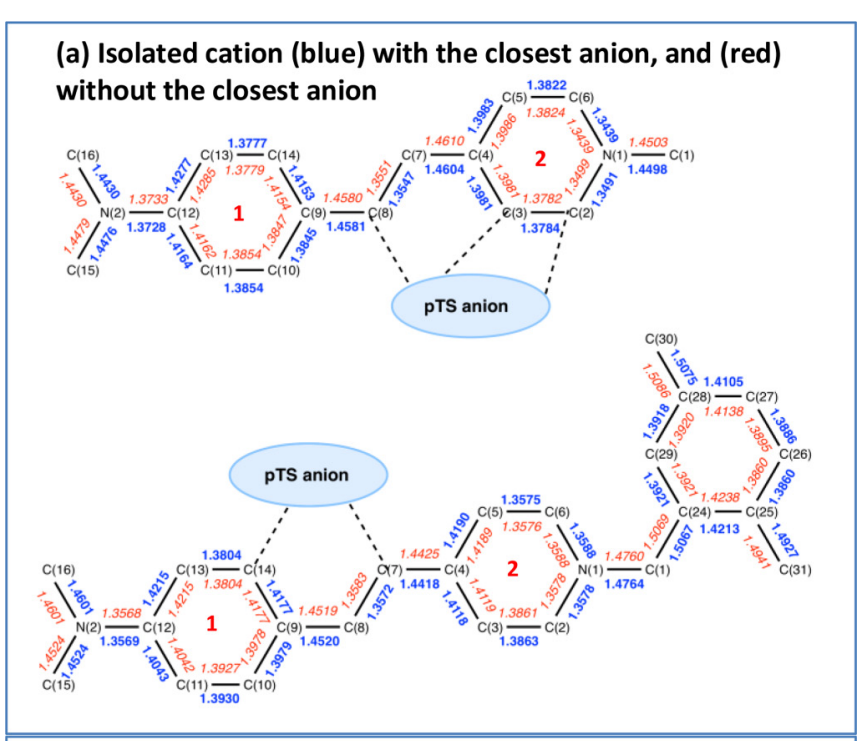

(b) Anion and cation with crystal field effects

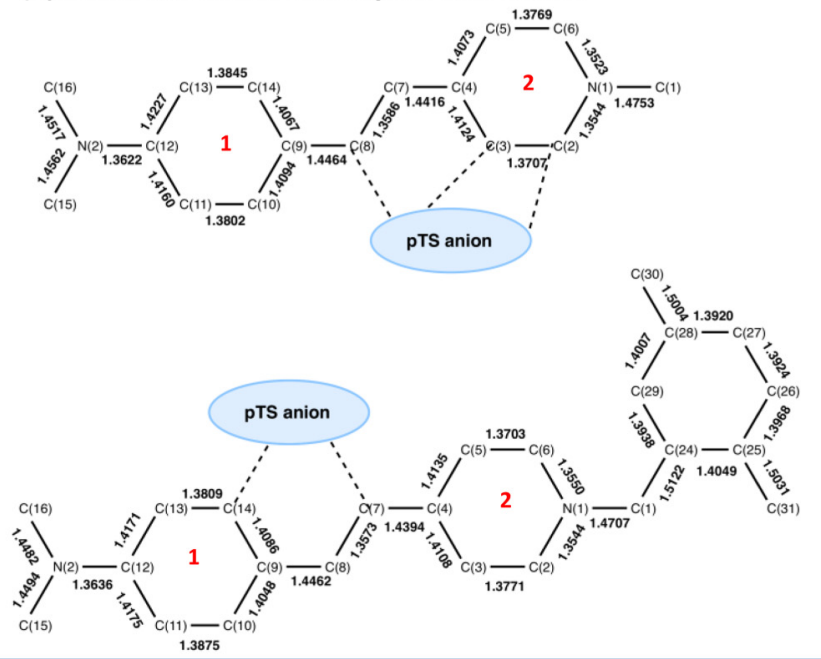

(c) Full crystal environment

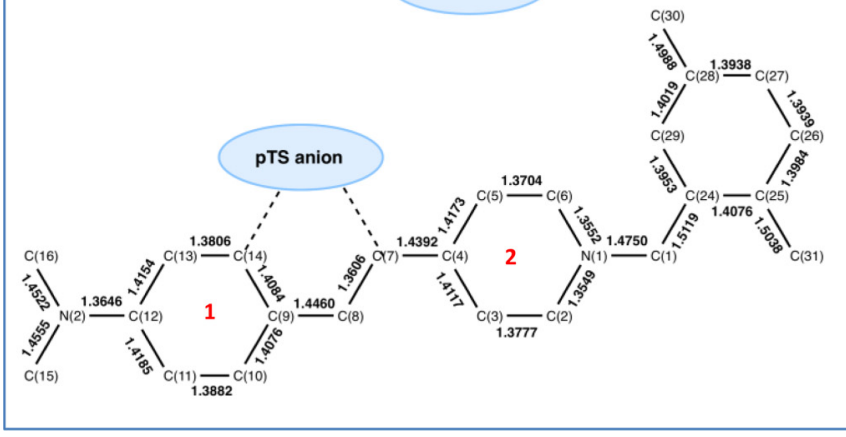

FIG. 3. Bond lengths for the cationic chromophore in DAST (top) and BP3 (bottom) for models (a)-(c). For model (a) the different colors indicate models with (blue) and without (red) the closest anion. crystal packing of the BP3 chromophore in the solid state, rather than present any significant electronic ICT contribution. Indeed, this moiety aligns perpendicular to the D- $\pi-A$ backbone of BP3; as such, its 1,2,4-trimethylbenzene group (dihedral angle: $\sim 93.7^{\circ}$ ) will preclude any effective ICT via the $\mathrm{C}(1)-\mathrm{C}(24)$ bonds, as confirmed by its respective bond lengths across all structural models. All of these findings corroborate those of Cole et al. [10] and thus benchmarks these data as having good accuracy.

\section{B. Intraionic charge-transfer and $\pi$-conjugation trends in cationic moieties of DAST and BP3}

\section{Bond-length descriptors and intramolecular charge transfer}

The strength-length relationship in chemical bonding can be correlated to the extent of ICT and thus the second-order NLO response [3]. To this end, two bond-length descriptors were employed to analyze the ICT in cations of DAST and BP3: (i) bond-length averages and (ii) BLA. The ICT analysis was applied to three distinct carbon-based $\pi$-conjugated structural fragments of DAST and BP3 that are common to their cations: the quinoidal ring (ring 1), the nonheteroatomic part of ring 2 , and the bridge that connects them (as labeled In Fig. 1). Bond-length averages are simply the statistical mean of the bond lengths in a given structural fragment. BLA can be described in several different ways. It is described here in its simplistic form, i.e., the statistical mean of all bond-length differences between vicinal carbon-carbon bonds within a specified molecular fragment. In cases where a bond connects to multiple bonds, the smallest bond-length difference of all options available is employed; e.g., the bond-length difference involving a bond that projects from an aromatic ring, there being two bonds from the ring that connect to this bond; the overall BLA calculation contains such cases. This BLA formalism may be considered as the classical method of assessing the extent of delocalization ensuing within a $\pi$-conjugated fragment of a molecular chromophore. This has been used extensively to analyze D- $\pi$-A structural motifs in organic NLO materials to deduce the level of ICT and thus their inherent prospects for second-order NLO function $[3,15]$.

The intraionic charge transfer across cations of DAST and BP3 can be quantified in fine detail, by breaking down the bond length and BLA contributions into those of ring 1, the nonheteroatomic part of ring 2 , and the bridge. The average bond lengths for each molecular fragment, as well as their BLA values, were calculated for each structural model, as were the overall BLA values for the cations (see Tables I and II). Clear trends across the structural models are highlighted with arrow annotations.

\section{Bond-length alternation (BLA) analysis of BP3}

All the BLA values for the BP3 cation decrease monotonically, revealing that the structural behavior of the three molecular fragments is uniform and is representative of the entire $\mathrm{D}-\pi-\mathrm{A}$ motif in each cation. These results also demonstrate a high level of internal consistency, thus 
TABLE I. Average bond lengths in the cationic moieties of DAST and BP3.

\begin{tabular}{|c|c|c|c|c|c|c|}
\hline \multirow[b]{2}{*}{ Model } & \multicolumn{6}{|c|}{ Bond length averages in the cationic moiety $(\AA)$} \\
\hline & $\begin{array}{l}\text { DAST } \\
\text { ring } 1\end{array}$ & $\begin{array}{l}\mathrm{BP} 3 \\
\text { ring } 1\end{array}$ & $\begin{array}{l}\text { DAST } \\
\text { bridge }\end{array}$ & $\begin{array}{c}\text { BP3 } \\
\text { bridge }\end{array}$ & $\begin{array}{c}\text { DAST } \\
\text { 4C-ring2 }\end{array}$ & $\begin{array}{c}\text { BP3 } \\
\text { 4C-ring2 }\end{array}$ \\
\hline (a) (i) isolated cation & 1.4014 & 1.4024 & 1.4247 & 1.4176 & 1.3893 & 1.3936 \\
\hline (a) (ii) isolated cation influenced by closest anion & 1.4012 & 1.4025 & 1.4244 & 1.4170 & 1.3892 & 1.3937 \\
\hline (b) cation and anion with crystal field effects & 1.4033 & 1.4027 & 1.4155 & 1.4143 & 1.3918 & 1.3929 \\
\hline (c) cation and anion in full crystal lattice environment & 1.4048 & 1.4031 & 1.4159 & 1.4153 & 1.3926 & 1.3943 \\
\hline
\end{tabular}

corroborating the robustness of the four multiphase structural models detailed in Table II. This reduction in BLA from gas to solid-state is particularly marked between models (a) and (b), whereupon crystal field forces start to influence the cation; the BLA continues to diminish until model (c), when the cation is contained within its full crystal lattice environment. BLA changes in ring 1 of BP3 are modest, and their absolute BLA values are the lowest of the entire BP3 cation, irrespective of the model; ring 1 thus appears to present a highly delocalized nature. In stark contrast, the bridge exhibits the highest absolute BLA values irrespective of the model; indeed, the bond lengths of the bridge display a distinct tendency towards a single-double-single canonical form of bonding across all models. Nonetheless, its BLA values drop markedly with the onset, and thence progressive build-up, of crystal field forces, in common with ring 1 . The carbon-based component of ring 2 in BP3 shows a similar trend in reduction, but with much smaller absolute BLA values to the extent that they represent a ring with a high level of delocalization.

The overall BLA value for BP3 cations in the crystal lattice environment [model (c)] is striking, by virtue of it being a perfect BLA value for organic second-order NLO applications, according to the stipulations of valence effective Hamiltonian (VEH) models, which indicate that $\beta$ is maximized for molecules whose $|\mathrm{BLA}|$ modulus is 0.04 [57]. Table II shows that the overall BLA value for BP3 cations tends to this perfect value, as models progressively accommodate more intraionic interactions and proceed from the gas phase to the solid state. In other words, the progressive build-up of intraionic interactions in the D- $\pi$-A moiety of the BP3 cations optimizes its $\beta$ value, until its crystalline form manifests perfect second-order NLO prospects from a purely electronic perspective.

This BLA calculation does not consider any steric influences on $\beta$ that BP3 cations might issue beyond the D- $\pi-\mathrm{A}$ moiety. This perfect BLA value of the BP3 cation is unlikely to be deterred by any internal BLA contribution from the tosylate anion as this is deemed to contribute negligibly to $\beta$. However, cation $\cdots$ anion interactions can readily affect the overall $\beta$, as has been shown by Cole et al. [10]. Such interactions are considered implicitly for the D- $\pi$-A moiety of the $\mathrm{BP} 3$ cation in this demonstrated optimization of $\beta$, via the bond length changes caused by such interactions. However, electronic effects in the BP3 cation beyond the D- $\pi$-A pathway are not considered by the BLA diagnostics. Moreover, steric effects are neglected in the BLA analysis.

The effect of the two $\mathrm{N}$ heteroatoms and their substituents, and the associated positive charge on N(1) in BP3, which lie beyond its $\mathrm{D}-\pi$-A motif, are thus considered separately. The bond lengths of the ethylamino group (D) display conventional structural configurations across all models, as expected. However, the acceptor group, A, manifests in a more complicated fashion. Its positive charge on $\mathrm{N}(1)$ is stabilized by its adjoining 1,2,5-trimethylphenyl group, whose ring is essentially aromatic in nature. The extent of this aromaticity can in fact be calculated by a distinct BLA value that is computed specifically for this ring. This shows that its aromatic nature is enhanced as the BP3 cation transitions from the gas to the solid phase, manifesting a distinct drop in an already small BLA value [model (a) (i): 0.0199; (a) (ii): 0.0180] between the structure of the isolated cation, and models (b) and (c) that incorporate crystal field effects and whose BLA values [model (b): 0.0066; model (c): 0.0068] are so small that they approach the cyanine limit [57]. The high degree of ring delocalization is thus propelled by crystal field effects. Its ring current is nonetheless isolated by its bridge to $\mathrm{N}(1)$, increasingly so as the model progresses from an isolated cation structure to an embedding within its crystalline environment, vis $a$ vis the progressively larger single-bond character of the $\mathrm{C}(1)-\mathrm{C}(24)$ bond, whose length increases monotonically from 1.5069 to $1.5119 \AA$ from model (a) (i) to model (c) (Fig. 4). Meanwhile, the N(1)-C(1) bond maintains a characteristic $\sigma$-bond character across all models [58]. Indeed, this ring is out-of-plane with the D- $\pi$-A moiety of the BP3 cation, as previously noted. These models thus show that this

TABLE II. Bond-length-alternation (BLA) values and overall BLA for the cationic moieties of DAST and BP3.

\begin{tabular}{|c|c|c|c|c|c|c|c|c|}
\hline \multirow[b]{2}{*}{ Model } & \multicolumn{6}{|c|}{ Bond length alternation (BLA) in the cationic moiety $(\AA)$} & \multicolumn{2}{|c|}{ Overall BLA $(\AA)$} \\
\hline & $\begin{array}{l}\text { DAST } \\
\text { ring } 1\end{array}$ & $\begin{array}{c}\mathrm{BP} 3 \\
\text { ring } 1\end{array}$ & $\begin{array}{l}\text { DAST } \\
\text { bridge }\end{array}$ & $\begin{array}{c}\text { BP3 } \\
\text { bridge }\end{array}$ & $\begin{array}{c}\text { DAST } \\
\text { 4C-ring } 2\end{array}$ & $\begin{array}{c}\text { BP3 } \\
\text { 4C-ring } 2\end{array}$ & $\begin{array}{c}D-\pi-A \\
\text { of DAST }\end{array}$ & $\begin{array}{l}D-\pi-A \\
\text { of BP3 }\end{array}$ \\
\hline (a) (i) isolated cation & 0.0271 & 0.0220 & 0.1044 & 0.0889 & 0.0122 & 0.0313 & 0.0491 & 0.0428 \\
\hline (a) (ii) cation influenced by closest anion & 0.0269 & 0.0219 & 0.1046 & 0.0897 & 0.0120 & 0.0314 & 0.0490 & 0.0429 \\
\hline (b) cation \& anion \& crystal field effects & 0.0225 & 0.0192 & 0.0854 & 0.0855 & 0.0257 & 0.0265 & 0.0417 & 0.0407 \\
\hline (c) cation \& anion within crystal lattice & 0.0204 & 0.0194 & 0.0971 & 0.0820 & 0.0247 & 0.0288 & 0.0446 & 0.0400 \\
\hline
\end{tabular}


aromatic ring tends toward perfect delocalization and thus greater electronic isolation from the rest of the cation, as BP3 tends towards crystallization. Cole et al. [10] previously showed that this aromatic ring is involved in three $\mathrm{C}-\mathrm{H}$... O interactions; one of these will impact the BLA of its ring; the other two will not as they connect to the bridge between the rings; they could nonetheless still affect $\beta$.

\section{Bond-length Alternation (BLA) analysis of DAST}

The cation of DAST exhibits more variable bond-length characteristics than that of BP3. Its ring 1 exhibits average bond lengths and BLA trends that are akin to those of the BP3 cation. In contrast, the average bond lengths associated with its bridge are markedly longer (weaker) than those of BP3 for models (a) (i) and (a) (ii), i.e., when cations of DAST are not subjected to crystal field forces; their BLA values are correspondingly higher than those of the BP3 cation for these two models. However, these average bond lengths and BLA values drop markedly between models (a) and (b), to the extent that both of these bond- length metrics become essentially the same as the cognate values for model (b) of the BP3 cation. Model (c) shows a curious feature whereby the BLA value for the bridge in the cation of DAST returns to nearly the BLA values of the models in (a); its average bond length also increases, albeit slightly. This suggests that while crystal field forces modeled outside of a crystal lattice augment the ICT for DAST, the explicit incorporation of highly directed forms of crystal field forces such as hydrogen-bonding, held within a crystal lattice as per model (c), deters ICT. The trend observed for ring 2 in the cation of DAST is particularly striking, when set against that of BP3. The average bond lengths of the carbon component of ring 2 for the cation of DAST are shorter (stronger) than those of BP3 for model (a), which is entirely counterpoint to the situation observed for the bridging unit that is common to these cations. The BLA values for the carbon component of ring 2 are significantly lower for cations of DAST than BP3 for model (a), thus corroborating the verity of this trend. Moreover, these BLA values display entirely opposite trends with those for the cation of DAST diminishing with the removal of crystal field forces, while those of BP3 diminish as crystal field forces are introduced. The diminution of these BLA values for cations of DAST upon removing crystal field forces is such that those of model (a) come close to the cyanine limit. Given that ring 2 contains a positive charge on $\mathrm{N}(1)$, this striking difference between BP3 and DAST is likely to result, at least in part, from the much greater charge stabilization on $\mathrm{N}(1)$ by the electron donating benzyl rather than methyl substituents in BP3 and DAST, respectively. Another rationale for this striking difference could be the $\mathrm{C}-\mathrm{H} \cdots \mathrm{C}(\pi)$ interaction that is present in DAST but absent in BP3, as previously deduced by Cole et al. [10]; they highlighted this distinction in interionic interactions between DAST and BP3 as the key reason for their different second-order NLO prospects.

The overall BLA values for the cation of DAST naturally reflect the lack of clear monotonic trend between models (a) to (c) for the bridging unit given its high BLA value will dominate the overall BLA metric. The BLA values themselves indicate a decent level of ICT, and thus good second-order
NLO prospects, judging from their proximity to the ideal $\mathrm{BLA}=0.04 \AA$ value posed by $\mathrm{VEH}$ calculations [57]. Indeed, the results indicate that the cation of DAST becomes near ideal $(\mathrm{BLA}=0.0417 \AA$ ) when crystal field forces are introduced in model (b); however, this BLA is compromised once cations of DAST are incorporated into the crystal lattice environment of model (c). This suggests that while crystal field forces from essentially nondirectional electrostatic interionic interactions appear to augment ICT in the D- $\pi-A$ cationic moiety of DAST, directed interionic interactions such as hydrogen bonds deter ICT and thus the second-order NLO response in DAST. This surmise corroborates previous notions proposed by Cole et al. [10]. In contrast to BP3, the $\mathrm{D}-\pi-\mathrm{A}$ moiety of DAST is synonymous to its entire cation except for its methyl extremities. Therefore, the ICT from the cation of DAST is likely to represent its full $\beta$ contribution save for any possible effect of the few $\mathrm{C}-\mathrm{H}$...O hydrogenbonds that involve the methyl groups (see Cole et al. [10]); the cation of DAST has no large substituents beyond its D- $\pi$-A moiety to cause steric effects, in contrast to BP3.

\section{Second-order NLO activity: Comparison of the molecular hyperpolarizability determined by five independent methods}

Hyperpolarizability coefficients, $\beta$, that represent the second-order NLO response at the molecular scale, were then determined for DAST and BP3 in the gas, liquid, and solid state. Previously, their molecular hyperpolarizabilities in crystalline form $\left[\beta_{z z z}^{\text {cry }}\right.$ (crystal) $]$ had only been determined via their powder SHG efficiencies. Yet, electrostatic moments derived from experimental charge densities that are afforded by high-resolution x-ray diffraction studies have successfully been used to determine the molecular hyperpolarizability of a material in its single-crystal form [29,59-61]. This determination employs the empirical method derived by Robinson [16], adapted slightly by Higginbotham et al., cf. Eq. (1) [17]:

$$
\beta_{i j k}=\left(\frac{2}{n}\right)^{2}\left(Q_{x x} Q_{y y}+Q_{y y} Q_{z z}+Q_{x x} Q_{z z}\right) O_{x y z},
$$

where $Q_{i j}$ refers to the second Cartesian moment, $O_{i j k}$ to the third Cartesian moment, and $n$ to the number of electrons. Thus, the static hyperpolarizability, $\beta_{0}^{M M}$ (crystal), was determined for DAST and BP3 in the single-crystal form, using the multipolar moments (MM) of the charge-density models for DAST (TAAM exp) and BP3 (MM exp) (see the Supplemental Material for details) [14]. The determination of $\beta$ in a crystalline environment is both rare and industrially pertinent, given that this is the phase in which a range of key practical applications for DAST have been considered [8].

The ability to calculate optical properties from crystal structure information presents an opportunity to develop structure-property relationships on materials with a valuable internal consistency. This point is especially pertinent when considering that there are significant practical issues with the accuracy of optical experiments that evaluate $\beta$. Indeed, experimentally determined values of $\beta$ are renowned for their large errors in quantification (typically 10-30\%) [62]. HyperRayleigh scattering (HRS) is considered to produce the most reliable experimental values of $\beta$, [19] and so our calculations of $\beta$ presented herein are compared against HRS values for 
TABLE III. Summary of the molecular optical nonlinearity of DAST and BP3 characterized using different methods.

\begin{tabular}{lcc}
\hline \hline Units: $\times 10^{-30}$ esu & DAST & BP3 \\
\hline$\beta_{0}^{\text {DFT }}($ gas $)$ & 159 & 194 \\
$\beta_{0}^{\mathrm{XWR}}($ gas + crystal field $)$ & 116 & 194 \\
$\beta_{0}^{\mathrm{HRS}}(\text { solution })^{\mathrm{a}}$ & $120 \pm 15^{\mathrm{b}}$ & $180 \pm 20^{\mathrm{c}}$ \\
$\beta_{z z z}^{\text {cry }}(\text { crystal })^{\mathrm{d}}$ & $37 \pm 10$ & $100-120$ \\
$\beta_{0}^{M M}$ (crystal) & 17 (unrefined TAAM) 43 (monopole refined) & 109 \\
\hline \hline
\end{tabular}

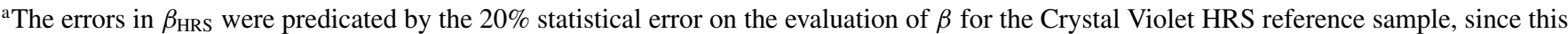
error propagates through to the uncertainty in $\beta$.

${ }^{\mathrm{b}} \mathrm{HRS}$ value previously reported by Teshome et al. [18].

${ }^{\mathrm{c}} \mathrm{HRS}$ value previously reported by Cole et al. [10].

${ }^{\mathrm{d}}$ Value estimated from the respective reported powder SHG efficiency: DAST [63] BP3 [11]. $c f$. SI, §S.4 for further details on the derivation of these values.

DAST [18] and BP3 [10]. That said, $\beta_{0}^{\mathrm{HRS}}$ values from HRS are determined on samples in solution. It is therefore prudent to theoretically and experimentally determine $\beta$ in a number of phases (in the gas-phase, solution, and the solid-state if possible), and place considerable importance on comparative trends in $\beta$ across various states of matter. Thus, DFT was used to calculate $\beta$ in the gas phase $\left[\beta_{0}^{\mathrm{DFT}}(\mathrm{gas})\right]$. Multiphase evaluations of both electron-density mappings and $\beta$ also allow the effects of interionic interactions to be distinguished, especially those pertaining to crystal-field effects. Thus, our theoretically generated electron-density models that were tempered against the experimentally determined models of DAST and BP3 using XWR, were also used to calculate $\beta$ values, $\beta_{0}^{\mathrm{XWR}}$ (gas + crystal field); these pertain to $\beta$ models that incorporate isotropic crystal field forces but not directional forces such as hydrogen bonds, which are only present in the models of DAST and BP3 cations held within their full crystal lattice environment.

The molecular hyperpolarizability values for DAST and BP3 that were derived from these five independent methods are shown in Table III.

Concerning the charge-density derivations of the molecular hyperpolarizabilities in the crystalline state generated by this work, the $\beta_{0}^{M M}$ value for BP3 is in excellent agreement with the $\beta_{z z z}^{\text {cry }}$ value obtained from the measured powder SHG efficiency, while $\beta_{0}^{M M}$ and $\beta_{z z z}^{\text {cry }}$ are in general agreement for DAST. The reader is directed to Sec. S.4 of the Supplemental Material for a detailed explanation of the derivation of the $\beta_{z z z}^{\text {cry }}$ values. Table III permits a cross comparison of the $\beta$ values of DAST and BP3, derived using the five independent methods, from which it is clear that the effects of crystal lattice packing impede both DAST and BP3 from achieving their maximum potential as second-order NLO-active chromophores. This is particularly true for DAST, where a reduction of $\sim 73 \%$ is observed between the gas phase (DFT) and the solid state, compared to a much lower reduction of $44 \%$ for BP3 under analogous circumstances; indeed, $\beta$ for BP 3 barely drops until it is incorporated into its crystal lattice environment. This is to be anticipated when considered together with the assessments of Sec. II B, where the presence of short interionic interactions between the respective cations and anions in DAST cause a significant reduction in ICT once cations of DAST were incorporated into a crystal lattice environment. In comparison, the D- $\pi$-A motif of BP3 presents a perfect BLA value in its crystal lattice environment. It can nonetheless be affected by steric effects and cation $\cdots$ anion interactions that lie beyond this D- $\pi$-A motif in the BP3 cation, which form on crystallization as noted in Sec. II B; thus, a significant drop in $\beta$ for BP3 is only observed upon forming its crystalline state. This diminution is much less in BP3 than in DAST owing to the lack of $\mathrm{C}-\mathrm{H} \cdots \mathrm{C}(\pi)$ interionic interactions in BP3, in contrast to DAST; thereby, the concerted presence of $\mathrm{C}-\mathrm{H} \cdots \mathrm{C}(\pi)$ and $\mathrm{C}-\mathrm{H} \cdots \mathrm{O}$ cation $\cdots$ anion interactions in DAST derivatives has been found to deter their second-order NLO prospects considerably [10]. Furthermore, the $\mathrm{C}-\mathrm{H} \cdots \mathrm{O}$ cation $\cdots$ anion interactions that are present in BP3 that lie beyond its $\mathrm{D}-\pi-\mathrm{A}$ motif include its shortest such interaction (see Cole et al. [10]); its perfect BLA value in the crystal lattice environment is thus somewhat protected from cation ...anion interactions.

Table III reveals that the molecular hyperpolarizability for DAST also drops considerably between the gas and solution phase; in stark contrast to BP3, which remains the same within error. This reduction of $\beta$ for DAST is likely to be a consequence of the possibility of forming deleterious $\mathrm{C}-\mathrm{H} \cdots \mathrm{C}(\pi)$ and $\mathrm{C}-\mathrm{H} \cdots \mathrm{O}$ cation $\cdots$ anion contacts once DAST is surrounded by a medium (crystal field forces for the XWR model, or a solution for the experimental results). The $\beta$ value for the $\mathrm{XWR}$, model (b), in DAST is thus the same as $\beta$ determined by experiment in solution within experimental error. The directional nature of crystal field forces will be minimal in this XWR model formalism; so, it captures interionic interactions in a similarly subtle and nondirection specific fashion to solution-based results. The full directional nature of the cation $\cdots$ anion interionic interactions will only be felt in model (c) which explicitly incorporates the influence of the crystal lattice environment; thus, $\beta$ for DAST drops heavily again between the solution and solid-state phase results.

On a technical point, there are two $\beta_{0}^{M M}$ (crystal) values given for DAST. $\beta_{0}^{M M}$ for DAST, as derived from the TAAM exp model, is perhaps a little underestimated, being just at the mark of $2 \sigma$ error, $c f .\left(17 \times 10^{-30} \mathrm{esu}\right)$ compared to $(37 \pm 10) \times 10^{-30}$ esu for $\beta_{z z z}^{\text {cry }}$. This is unsurprising given the limited accuracy of a TAAM model, especially that which pertains to the multipolar population of its atoms. Indeed, the value of $\beta_{0}^{M M}$ is deduced using the equation of Higginbotham et al. [17], which requires the determination of quadrupolar and octupolar atomic moments using Stockholder spatial partitioning [64]; so incorrect multipolar populations will have a significant impact on the accurate determination of $\beta_{0}^{M M}$. As a consequence of the unusual $\beta_{0}^{M M}$ result for DAST determined 
by the TAAM exp model, and given the importance of correctly assigned multipolar populations, an attempt was made to refine all monopole populations against the $\mathrm{x}$-ray data of DAST, while all the other higher-order multipolar terms were kept unrefined; the limiting resolution of the x-ray data mandated this restriction to a monopolar model for charge-density analysis. The $\beta_{0}^{M M}$ value $\left(43 \times 10^{-30} \mathrm{esu}\right)$ derived from this modified TAAM exp model of DAST is a bit more in line with the $\beta_{z z z}^{\text {cry }}$ value, confirming the importance of the accurate assignment of multipolar populations in the undertaking of a Stockholder spatial partitioning of the molecular charge density of a molecule.

\section{CONCLUSIONS AND FUTURE RESEARCH}

This extensive multiphase analysis of the most commercially significant ionic NLO chromophore, DAST, and one of its derivatives, BP3, has demonstrated an innovative approach for studying the crystalline environment of NLO chromophores in a piecewise fashion. Thereby, the intraionic and interionic interactions in DAST and its derivative, BP3, have been related to their second-order NLO function at the molecular scale, and across multiple phases via four structural models. BLA analysis has revealed distinct intraionic trends in terms of the ICT and thus second-order NLO capabilities of the D- $\pi$-A motifs in DAST and BP3 across the gas, \{ gas + crystal field forces $\}$, solution, and crystalline states of matter. The D- $\pi$-A motif for BP3 tends to a perfect BLA value upon crystallization. Better stabilization of its positively charged nitrogen by its $\mathrm{N}$-substituted benzyl group, relative to the corresponding methyl group on DAST, helps towards achieving this perfect BLA value for BP3. The $\beta$ value of BP3 is essentially constant in all phases except for the crystalline state, where it appears to be deterred via $\mathrm{C}-\mathrm{H} \cdots \mathrm{O}$ cation $\cdots$ anion interactions that are not associated with the D- $\pi$-A constituent of the BP3 cation. In stark contrast, the $\beta$ value for DAST is progressively deterred from the gas phase to its crystalline form. This multiphase deleterious effect in DAST appears to be primarily a consequence of its manifestation of both $\mathrm{C}-\mathrm{H} \cdots \mathrm{C}(\pi)$ and $\mathrm{C}-\mathrm{H} \cdots \mathrm{O}$ cation $\cdots$ anion interactions that have been shown previously to deter DAST derivatives [10]. The lower ability of DAST to stabilize positive charge on the nitrogen of its pyridinium constituent, as opposed to BP3 whose positive charge is stabilized by a large benzyl side group, may also be a factor in this difference between DAST and BP3; indeed, their opposite BLA trends observed in ring 2 were found to deter the overall BLA value of the D- $\pi$-A motif for DAST. The molecular hyperpolarizability, $\beta$, has been derived via the $\{$ gas + crystal field forces $\}$ and solid-state structural models; they have been compared to reported experimental values of $\beta$ measured in solution or in a powder, as well as gas-phase ab initio DFT calculations. The $\beta$ values follow the same structural trends as those observed for the competition between intraionic and interionic interactions in BP3 and DAST, thus rationalizing their respective second-order NLO origins at the molecular scale. This is exciting both scientifically and technically; technically because this study presents a rare application of multiphase determinations of $\beta$, with the especially scarce evaluations of $\beta$ in the crystalline state derived solely from $\mathrm{x}$-ray diffraction data [17], and in the gas state with the influence of crystal field forces incorporated using XWR $[43,65,66]$ with all results comparing very nicely. Scientifically, these results not only corroborate our previous findings that certain cation $\cdots$ anion interactions dictate the second-order NLO function of DAST and its derivatives; they also afford newfound structure-property relationships and an associated knowledge base whose fine detail and multiphase information about DAST-based compounds is primed for the molecular engineering of new DAST derivatives with better tailored second-order NLO properties.

In particular, the model constructs demonstrated via this work, using high-resolution $\mathrm{x}$-ray diffraction data for the purposes of validation, can now be applied to low-resolution $\mathrm{x}$-ray diffraction data. Indeed, low-resolution x-ray diffraction data have already been used successfully to model nonionic organic NLO materials, more generally, by concerting XWR with structure factors from multipolar structural models of these experimental data [67]. Enabling this lower requirement of low-resolution x-ray diffraction data opens up a much wider range of materials for study, using these methods. This is because such experimental data are commonplace in repositories such as the Cambridge Structural Database [68]. The ability to employ this much greater databank of knowledge with model constructs based on those developed herein will allow this area of research to extend toward large-scale data-driven NLO materials prediction. This is very timely, now that there is a greater accessibility of high-performance computing and a growing importance of big data.

\section{ACKNOWLEDGMENTS}

J.M.C. is grateful for the BASF/Royal Academy of Engineering Research Chair in Data-Driven Molecular Engineering of Functional Materials, which is partly supported by the STFC via the ISIS Neutron and Muon Facility. J.M.C. also thanks the 1851 Royal Commission of the Great Exhibition for the 2014 Design Fellowship, hosted by Argonne National Laboratory where DOE Office of Science, Office of Basic Energy Sciences, supported work done under Contract No. DE-AC02-06CH11357. T.-C.L. acknowledges the Taiwanese Government for a Studying Abroad Scholarship. C.M.A. is indebted to the EPSRC, UK, for a DTA Ph.D. studentship (Grants No. EP/J500380/1 and No. EP/L504920/1). The authors thank Prof. Dr. S. Grabowsky, Institute of Inorganic Chemistry and Crystallography, University of Bremen, Germany for access to computational facilities and supervisorial support for L.A.M. O.-P.K. is grateful for the support by the National Research Foundation of Korea (NRF) funded by the Ministry of Science, ICT \& Future Planning, Korea (No. 2014R1A5A1009799 and No. 2016R1A2B4011050). All authors thank the EPSRC UK National Service for Computational Chemistry Software (NSCCS), EP/J003921/1, and acknowledge contributions from its staff in supporting this work.

J.M.C. conceived the study. O.-P.K. and S.-C.L. provided the crystal samples. T.-C.L. reduced the experimental data under the supervision of J.M.C. C.M.A. and T.-C.L. carried out $a b$ initio calculations; T.-C.L. generated DenProp models. T.-C.L. conducted the multipolar refinements that were used by C.M.A to undertake topological analysis. C.M.A carried out the x-ray wave function refinements; all 
calculations by C.M.A. and T.-C.L. were supervised by J.M.C. L.A.M. carried out the initial Hirshfeld Atom Refinement of DAST, and advised C.M.A. on the application of XWR to ionic systems. C.M.A. and J.M.C. interpreted the results and drafted the paper with input from all authors. All authors have given approval to the final version of the manuscript.

The authors declare no competing financial interest.
[1] L. T. Cheng, W. Tam, S. H. Stevenson, G. R. Meredith, G. Rikken, and S. R. Marder, J. Phys. Chem. 95, 10631 (1991).

[2] L. T. Cheng, W. Tam, S. R. Marder, A. E. Stiegman, G. Rikken, and C. W. Spangler, J. Phys. Chem. 95, 10643 (1991).

[3] J. M. Cole, Philos. Trans. R. Soc. London Ser. Math. Phys. Eng. Sci. 361, 2751 (2003).

[4] J. L. Oudar and J. Zyss, Phys. Rev. A 26, 2016 (1982).

[5] S. R. Marder, J. W. Perry, and C. P. Yakymyshyn, Chem. Mater. 6, 1137 (1994).

[6] F. Pan, M. S. Wong, C. Bosshard, and P. Günter, Adv. Mater. 8, 592 (1996).

[7] S. R. Marder, J. W. Perry, and W. P. Schaefer, Science 245, 626 (1989).

[8] M. Jazbinsek, L. Mutter, and P. Gunter, IEEE J. Sel. Top. Quantum Electron. 14, 1298 (2008).

[9] Y. Takahashi, H. Adachi, T. Taniuchi, M. Takagi, Y. Hosokawa, S. Onzuka, S. Brahadeeswaran, M. Yoshimura, Y. Mori, H. Masuhara, T. Sasaki, and H. Nakanishi, J. Photochem. Photobiol. Chem. 183, 247 (2006).

[10] J. M. Cole, T.-C. Lin, A. J. Edwards, R. O. Piltz, G. Depotter, K. Clays, S.-C. Lee, and O.-P. Kwon, ACS Appl. Mater. Interfaces 7, 4693 (2015).

[11] P.-J. Kim, J.-H. Jeong, M. Jazbinsek, S.-J. Kwon, H. Yun, J.-T. Kim, Y. S. Lee, I.-H. Baek, F. Rotermund, P. Günter, and O.-P. Kwon, Cryst. Eng. Commun. 13, 444 (2010).

[12] J. Kim, O.-P. Kwon, M. Jazbinsek, Y. C. Park, J.-I. Seo, and Y. S. Lee, J. Phys. Chem. C 115, 23535 (2011).

[13] S. Follonier, M. Fierz, I. Biaggio, U. Meier, C. Bosshard, and P. Günter, JOSA B 19, 1990 (2002).

[14] See Supplemental Material at http://link.aps.org/supplemental/ 10.1103/PhysRevMaterials.4.115203 for details of the experimental and computational methods used, multipolar refinements, residual electron density maps of the ions, full tabulation of the topological analysis, and XWR validation tests.

[15] S. R. Marder, C. B. Gorman, B. G. Tiemann, J. W. Perry, G. Bourhill, and K. Mansour, Science 261, 186 (1993).

[16] F. N. H. Robinson, Bell Syst. Tech. J. 46, 913 (1967).

[17] A. P. Higginbotham, J. M. Cole, M. A. Blood-Forsythe, and D. D. Hickstein, J. Appl. Phys. 111, 033512 (2012).

[18] A. Teshome, M. D. H. Bhuiyan, G. J. Gainsford, M. Ashraf, I. Asselberghs, G. V. M. Williams, A. J. Kay, and K. Clays, Opt. Mater. 33, 336 (2011).

[19] K. Clays and A. Persoons, Phys. Rev. Lett. 66, 2980 (1991).

[20] F. L. Hirshfeld, Acta Crystallogr. Sect. A 32, 239 (1976).

[21] R. F. W. Bader, Acc. Chem. Res. 18, 9 (1985).

[22] R. F. Stewart, E. R. Davidson, and W. T. Simpson, J. Chem. Phys. 42, 3175 (1965).

[23] E. Espinosa, I. Alkorta, J. Elguero, and E. Molins, J. Chem. Phys. 117, 5529 (2002)

[24] E. Espinosa, M. Souhassou, H. Lachekar, and C. Lecomte, Acta Crystallogr. B 55, 563 (1999).
[25] E. Espinosa, E. Molins, and C. Lecomte, Chem. Phys. Lett. 285, 170 (1998).

[26] C. Gatti, E. May, R. Destro, and F. Cargnoni, J. Phys. Chem. A 106, 2707 (2002).

[27] S. C. Abrahams and E. T. Keve, Acta Crystallogr. A 27, 157 (1971).

[28] J. Henn and K. Meindl, Int. J. Mater. Chem. Phys. 1, 417 (2016).

[29] J. M. Cole, R. C. B. Copley, G. J. McIntyre, J. A. K. Howard, M. Szablewski, and G. H. Cross, Phys. Rev. B 65, 125107 (2002).

[30] T. O. Lopes, D. F. S. Machado, C. Risko, J.-L. Brédas, and H. C. B. de Oliveira, J. Phys. Chem. Lett. 9, 1377 (2018).

[31] G. M. Sheldrick, Acta Crystallogr. A 64, 112 (2008).

[32] L. J. Farrugia, J. Appl. Crystallogr. 45, 849 (2012).

[33] A. Ø. Madsen, J. Appl. Crystallogr. 39, 757 (2006).

[34] R. H. Blessing, Acta Crystallogr. B 51, 816 (1995).

[35] A. Volkov, M. Messerschmidt, and P. Coppens, Acta Crystallogr. D 63, 160 (2007).

[36] A. Volkov, X. Li, T. Koritsanszky, and P. Coppens, J. Phys. Chem. A 108, 4283 (2004).

[37] P. M. Dominiak, A. Volkov, X. Li, M. Messerschmidt, and P. Coppens, J. Chem. Theory Comput. 3, 232 (2007).

[38] A. Volkov, P. Macchi, L. J. Farrugia, C. Gatti, C. Mallinson, T. Richter, and T. Koritsanszky, XD2006 - a Computer Programfor Multipole Refinement, Topological Analysis of Charge Densities and Evaluation of Intermolecular Energies from Experimental or Theoretical Structure Factors (2006).

[39] W. Kohn and L. J. Sham, Phys. Rev. 140, A1133 (1965).

[40] P. Hohenberg and W. Kohn, Phys. Rev. 136, B864 (1964).

[41] M. J. Frisch, G. W. Trucks, H. B. Schlegel, G. E. Scuseria, M. A. Robb, J. R. Cheeseman, G. Scalmani, V. Barone, B. Mennucci, G. A. Petersson, H. Nakatsuji, M. Caricato, X. Li, H. P. Hratchian, A. F. Izmaylov, J. Bloino, G. Zheng, J. L. Sonnenberg, M. Hada, M. Ehara, K. Toyota, R. Fukuda, J. Hasegawa, M. Ishida, T. Nakajima, Y. Honda, O. Kitao, H. Nakai, T. Vreven, J. A. Montgomery Jr, J. E. Peralta, F. Ogliaro, M. Bearpark, J. J. Heyd, E. Brothers, K. N. Kudin, V. N. Staroverov, R. Kobayashi, J. Normand, K. Raghavachari, A. Rendell, J. C. Burant, S. S. Iyengar, J. Tomasi, M. Cossi, N. Rega, N. J. Millam, M. Klene, J. E. Knox, J. B. Cross, V. Bakken, C. Adamo, J. Jarmillo, R. Gomperts, R. E. Stratmann, O. Yazyev, A. J. Austin, R. Cammi, C. Pomelli, J. W. Ochterski, R. L. Martin, K. Morokuma, V. G. Zakrzewski, G. A. Voth, P. Salvador, J. J. Damnenberg, S. Dapprich, A. D. Daniels, Ö. Farkas, J. B. Foresman, J. V. Ortiz, J. Cioslowski, and D. J. Fox, Gaussian09 (Gaussian, Inc., Wallingford CT, 2009).

[42] A. Volkov, T. Koritsanszky, M. Chodkiewicz, and H. F. King, J. Comput. Chem. 30, 1379 (2009).

[43] M. Woińska, D. Jayatilaka, B. Dittrich, R. Flaig, P. Luger, K. Woźniak, P. M. Dominiak, and S. Grabowsky, Chem. Phys. Chem. 18, 3334 (2017).

[44] S. C. Capelli, H.-B. Bürgi, B. Dittrich, S. Grabowsky, and D. Jayatilaka, IUCrJ 1, 361 (2014). 
[45] D. Jayatilaka, Phys. Rev. Lett. 80, 798 (1998).

[46] D. Jayatilaka and D. J. Grimwood, Acta Crystallogr. A 57, 76 (2001).

[47] D. Jayatilaka and D. J. Grimwood, in Computer Science -ICCS 2003, edited by P. M. A. Sloot, D. Abramson, A. V. Bogdanov, Y. E. Gorbachev, J. J. Dongarra, and A. Y. Zomaya (Springer, Berlin, 2003), pp.142-151.

[48] F. Weigend and R. Ahlrichs, Phys. Chem. Chem. Phys. 7, 3297 (2005).

[49] D. Jayatilaka and B. Dittrich, Acta Crystallogr. A 64, 383 (2008).

[50] T. Lu and F. Chen, J. Comput. Chem. 33, 580 (2012).

[51] D. Jayatilaka, P. Munshi, M. J. Turner, J. A. K. Howard, and M. A. Spackman, Phys. Chem. Chem. Phys. 11, 7209 (2009).

[52] Rigaku, CrystalClearTM 2.0 (Rigaku (Europe), Sevenoaks, Kent TN15 6QY, England, n.d.).

[53] R. H. Blessing, Acta Crystallogr. A 51, 33 (1995).

[54] R. H. Blessing, J. Appl. Crystallogr. 30, 421 (1997).

[55] G. M. Sheldrick, XPREP in SHELXTL (Siemens Analytical xray Instruments, Inc., Madison, Wisconsin, 1995).

[56] X.-M. Duan, S. Okada, H. Oikawa, H. Matsuda, and H. Nakanishi, Jpn. J. Appl. Phys. 33, L1559 (1994).

[57] S. Dähne and R. Radeglia, Tetrahedron 27, 3673 (1971).
[58] F. H. Allen, O. Kennard, D. C. Watson, L. Brammer, A. G. Orpen, and R. Taylor, J. Chem. Soc. Perkin Trans. 2, S1 (1987).

[59] J. M. Cole, A. E. Goeta, J. A. K. Howard, and G. J. McIntyre, Acta Crystallogr. B 58, 690 (2002).

[60] J. M. Cole, T.-C. Lin, C. M. Ashcroft, J. Perez-Moreno, Y. Tan, P. Venkatesan, A. P. Higginbotham, P. Pattison, A. J. Edwards, R. O. Piltz, K. Clays, and A. Ilangovan, J. Phys. Chem. C 120, 29439 (2016)

[61] T.-C. Lin, J. M. Cole, A. P. Higginbotham, A. J. Edwards, R. O. Piltz, J. Pérez-Moreno, J.-Y. Seo, S.-C. Lee, K. Clays, and O.-P. Kwon, J. Phys. Chem. C 117, 9416 (2013).

[62] M. G. Kuzyk and C. W. Dirk (eds.), Characterization Techniques and Tabulations for Organic Nonlinear Optical Materials (Marcel Dekker, New York, 1998).

[63] U. Meier, M. Bösch, C. Bosshard, F. Pan, and P. Günter, J. Appl. Phys. 83, 3486 (1998).

[64] F. L. Hirshfeld, Theor. Chim. Acta 44, 129 (1977).

[65] D. D. Hickstein, J. M. Cole, M. J. Turner, and D. Jayatilaka, J. Chem. Phys. 139, 064108 (2013).

[66] J. M. Cole and D. D. Hickstein, Phys. Rev. B 88, 184105 (2013).

[67] C. M. Ashcroft, J. M. Cole, E. A. Boardman, T.-C. Lin, J. PerezMoreno, and K. Clays, J. Phys. Chem. C 123, 665 (2019).

[68] F. H. Allen, Acta Crystallogr. B 58, 380 (2002). 